\title{
Prevención de recurrencia de infección por hepatitis $B$ en receptores de trasplante hepático utilizando dosis bajas de inmunoglobulina antihepatitis B y análogo de nucleósido
}

\section{Prevention of Hepatitis B Recurrence in Liver Transplant Recipients Using Low Doses of Anti-Hepatitis B Immunoglobulin and Nucleoside Analogue}

Diana Moncada, MD, ${ }^{1}$ Octavio Muñoz, MD, ${ }^{2}$ Salomón Daguer, MD, ${ }^{1}$ Óscar Santos, MD, ${ }^{2}$ Juan Ignacio Marín, MD, ${ }^{2}$ Sergio Hoyos, MD, MSc, ${ }^{2}$ Carlos Guzmán, MD, ${ }^{3}$ Álvaro Mena, MD, ${ }^{3}$ Juan Carlos Restrepo, MD, MSc, PhD. ${ }^{2}$

Medicina Interna, Universidad Pontificia Bolivariana. Medellín, Colombia.

Grupo de Gastrohepatología, Universidad de Antioquia. Facultad de Medicina, Universidad de Antioquia. Hospital "Pablo Tobón Uribe". Medellín, Colombia.

3 Facultad de Medicina, Universidad de Antioquia. Hospital "Pablo Tobón Uribe”. Medellín, Colombia.

\begin{abstract}
Resumen
Introducción: la hepatitis B es una causa importante de trasplante hepático; produce 1 millón de muertes anuales. El uso de inmunoglobulina antihepatitis $B$ en dosis altas y análogos de nucleósidos redujeron en un $90 \%$ la reinfección del injerto. Objetivo: evaluar la eficacia de dosis bajas de inmunoglobulina postrasplante para prevenir la reinfección del injerto. Metodología: serie de casos retrospectiva de pacientes trasplantados en el Hospital "Pablo Tobón Uribe", entre enero de 2004 y septiembre de 2014, que recibieron inmunoglobulina después del trasplante. Se evaluaron la carga viral de hepatitis $B$, las transaminasas y los marcadores serológicos para documentar recaída, además de variables como mortalidad, complicaciones, disfunción del injerto, reacciones adversas y costos. Resultados: fueron 18 pacientes trasplantados con hepatitis B; 50\% tenía hepatocarcinoma; $22 \%$, cirrosis; y $22 \%$, insuficiencia hepática aguda. La mediana de seguimiento fue de 43,27 meses (14,7-65,2). De los pacientes, 2 tuvieron antígeno de superficie positivo en el postrasplante y en 1 hubo recaída con carga viral positiva a los 41 meses. La tasa de reinfección del injerto fue del 5,5\%. No hubo muertes. Se estimó que el costo de usar dosis bajas de inmunoglobulina fue menor comparado con las dosis altas a 6 meses de terapia; sin embargo, no se hizo estudio de costo-efectividad. La disfunción del injerto fue del 10\% a 33 meses. Conclusión: con dosis bajas de inmunoglobulina se previno la reinfección del injerto, similar a lo reportado en otras series. Mientras los esquemas libres de inmunoglobulina logran demostrar su utilidad a largo plazo, usar dosis bajas de inmunoglobulina sigue siendo útil.
\end{abstract}

Palabras clave

Trasplante de hígado, hepatitis B, inmunoglobulina, antiviral.

\begin{abstract}
Introduction: Hepatitis B results in one million deaths every year and is is an important reason for liver transplantation. The use of anti-hepatitis B immunoglobulin at high doses and nucleoside analogues have reduced reinfection of the graft by $90 \%$. Objective: This study evaluated the efficacy of low doses of immunoglobulin to prevent reinfection of grafts after transplantation. Methodology: This is a retrospective study of a series of patients who had been transplanted and who received immunoglobulin after transplantation at the Hospital Pablo Tobón Uribe between January 2004 and September 2014. Hepatitis B viral load, transaminase and serological markers were used to document relapses. Other variables studied included mortality, complications, graft dysfunction, adverse reactions and costs. Results: There were 18 patients with hepatitis B who had transplants: 50\% had hepatocarcinoma, 22\% had cirrhosis, and $22 \%$ had acute liver failure. The median follow-up time was 43.27 months with a range of 14.7 to 65.2 months. Two patients tested positive for surface antigen in the post-transplant period and one relapsed and had a positive viral load at 41 months. The graft reinfection rate was $5.5 \%$. There were no deaths. It was estimated that the cost of using low doses of immunoglobulin was lower than that of using high doses at 6 months of therapy, but no cost-effectiveness study was done. Graft dysfunction was $10 \%$ to 33 months. Conclusion: Low doses of immunoglobulin prevented reinfection of grafts in a way that is similar to that reported in other series. While immunoglobulin free schemes have proven to be useful for the long term, low doses of immunoglobulin remain useful.
\end{abstract}

Key words

Liver transplantation, hepatitis B, immunoglobulin, antiviral. 


\section{INTRODUCCIÓN}

La hepatitis B es una infección común en el mundo. Cerca de 2 billones de personas han tenido contacto con el virus y 400000 tienen hepatitis B crónica (1). Colombia es un país de endemicidad intermedia (2), cuya incidencia global fue de 4,36 casos/ 100000 habitantes a noviembre de 2014 . Hay regiones de mayor incidencia como Amazonas, Norte de Santander, Guaviare y Chocó, con 23,8, 12,5, 9,13 y 8,89 casos/100 000 habitantes, respectivamente (3).

Anualmente en el mundo ocurren 600000 muertes por cirrosis o carcinoma hepatocelular a consecuencia de la infección crónica, además de insuficiencia hepática aguda por infección reciente o reactivación en portadores crónicos. La infección crónica por virus B da cuenta del 10\% de las indicaciones de trasplante hepático $(\mathrm{TOH})(1)$, pero la reinfección del injerto es un desenlace factible.

Sin profilaxis antiviral postrasplante, la infección del injerto ocurre entre el $75 \%$ y el $80 \%$ de los casos, con una mortalidad del $50 \%$ a 2 años (4). Con el uso de profilaxis antiviral, la recurrencia se disminuye al 10\% (1).

La inmunoglobulina antihepatitis B (HBIG) en dosis altas (10 $000 \mathrm{UI}$ intravenosa [IV]/mensual) fue una de las primeras estrategias de profilaxis utilizadas para disminuir la infección del injerto entre un $20 \%$ y un $35 \%$ (5), por lo que la reinfección ocurre en más de la mitad de los casos debido al surgimiento de virus con mutantes de escape del antígeno de superficie (HBsAg) (6). Después, la lamivudina mostró mejor eficacia comparada con la HBIG a corto plazo (7); sin embargo, la lamivudina en monoterapia produjo altas tasas de resistencia, que llevaron a recaídas del $40 \%-50 \%$ (8) a 3 años. Ulteriormente la combinación de HBIG en dosis altas y lamivudina permitió reducir las recurrencias a menos del $10 \%$, con menor probabilidad de resistencia $(6,9)$; no obstante, este esquema significa altos costos en países en desarrollo. Para superar el problema del costo, se han empleado protocolos de dosis bajas de HBIG $400 \mathrm{UI} /$ intramusculares (IM) mensuales, que han demostrado efectividad similar a la evidenciada con dosis altas $(9,10)$. El objetivo de este estudio es describir la eficacia de la combinación de dosis bajas de HBIG IM y un análogo nucleósido para prevenir la reinfección del injerto hepático por virus de hepatitis B después del trasplante en el Hospital "Pablo Tobón Uribe" de la ciudad de Medellín, Colombia.

\section{MÉTODOS}

\section{Población}

Serie de casos retrospectiva. Se revisaron las historias clínicas de pacientes con infección por virus de hepatitis $B$ sometidos a trasplante hepático por insuficiencia hepática aguda, cirrosis con complicaciones y uno o varios focos de hepatocarcinoma atendidos en el Hospital "Pablo Tobón Uribe" (HPTU) entre enero de 2004 y septiembre de 2014, quienes recibieron profilaxis con HBIG y antiviral (entecavir, lamivudina o tenofovir) en el postrasplante. Se excluyeron los pacientes que recibieron menos de 7 días de HBIG, con seguimiento inferior a 6 meses o con datos insuficientes para evaluar el desenlace primario. Todos los pacientes tenían hepatitis B diagnosticada por HBsAg positivo y anticuerpos anti-core tipo $\operatorname{IgM}(\mathrm{HbcIgM})$ o tipo $\operatorname{IgG}$ (HbcIgG) antes del trasplante. Se evaluó la coinfección con virus de hepatitis $\mathrm{C}(\mathrm{HCV})$, hepatitis $\mathrm{D}(\mathrm{HDV})$ e inmunodeficiencia humana (VIH).

Se recolectaron variables clínicas y demográficas, tiempo de seguimiento, meses de uso de inmunoglobulina, disfunción del injerto (definida como la elevación de las transaminasas dos veces por encima del valor normal), mortalidad, serologías para $\mathrm{HBsAg}$, antígeno $e$ de hepatitis B $(\mathrm{HBeAg})$ pretrasplante, anticuerpos contra el antígeno $e$ (AntieHb) en el postrasplante, carga viral de hepatitis B (CV-VHB) pre y postrasplante, transaminasas, profilaxis utilizada e inmunosupresión a largo plazo.

\section{Profilaxis de reinfección}

Se realizó un esquema de dosis baja de HBIG con 400 UI/ IM diariamente por 7 días consecutivos, que inició en la fase anhepática y continuó con 400 UI IM mensual. Los primeros pacientes recibieron profilaxis indefinida con HBIG; ulteriormente se estableció una duración de 6 meses. Todos los pacientes recibieron además un análogo nucleósido oral indefinidamente. Quienes recibían lamivudina previo al trasplante continuaban con el mismo tratamiento si la carga viral al momento del trasplante era negativa; quienes no tenían tratamiento o tenían actividad replicativa viral recibieron entecavir $1 \mathrm{mg}$ cada día en el postrasplante. Los pacientes con carga viral positiva al momento del trasplante recibieron HBIG por más tiempo.

\section{Esquema de inmunosupresión}

Se aplicó el protocolo de inmunosupresión utilizado por el Grupo de Gastrohepatología, consistente en 1 gramo de metilprednisolona IV en el intraoperatorio y dosis descendentes hasta el sexto día; luego, $20 \mathrm{mg}$ de prednisolona cada día durante 3 meses. Los pacientes con insuficiencia renal o riesgo de presentarla recibieron micofenolato $1000 \mathrm{mg}$ cada 12 horas y aquellos con función renal normal o bajo riesgo de insuficiencia renal recibieron azatioprina $1-2 \mathrm{mg} / \mathrm{kg}$; el inhibidor de la calcineurina (ciclosporina o tacrolimús) se inició 
en las siguientes 6 a 18 horas, hasta las 72 horas postrasplante según la función renal. Todos los pacientes tuvieron seguimiento estricto por consulta externa.

\section{Seguimiento virológico}

Se hizo medición del HBsAg en el postrasplante. En quienes el resultado fue positivo, se llevó a cabo CV-VHB en tiempo real; también se realizó carga viral en los pacientes con elevación de las transaminasas. La reinfección del injerto fue definida como CV-VHB positiva en el postrasplante.

\section{Análisis estadístico}

La recolección y análisis de los datos se hizo en el software SPSS $^{\circ}$ versión 20 (SPSS Inc.; Chicago, Illinois, USA). Las variables cuantitativas se expresan como media o mediana, con sus respectivas medidas de dispersión según la distribución de la variable utilizando la prueba de Shapiro Wills; Se usaron curvas de supervivencia de Kaplan-Meier para evaluar la disfunción del injerto a largo plazo.

\section{Aspectos éticos}

El estudio fue aprobado por el Comité de Ética del Hospital "Pablo Tobón Uribe" y se ciñó a la resolución 008430 de 1993 del Ministerio de Salud de la República de Colombia sobre aspectos éticos de la investigación en seres humanos.

\section{RESULTADOS}

Se recolectaron 18 pacientes trasplantados con infección por virus de hepatitis B entre enero de 2004 y septiembre de 2014 y que cumplieron los criterios de inclusión. Las características basales de la población se describen en la tabla 1 . El 77,8\% de los pacientes eran hombres, con edad promedio de 52,6 $\pm 11,28$ años. El $83 \%$ de los pacientes tenía alguna comorbilidad, siendo la hipertensión arterial y la diabetes las más frecuentes en el $40 \%$ y $26,6 \%$ de los casos, respectivamente. La mediana del seguimiento fue de 43,27 meses $(14,7-65,2)$. Las indicaciones de trasplante fueron: $50 \%$, hepatocarcinoma; $22,2 \%$, cirrosis con complicaciones; y $22,2 \%$, insuficiencia hepática aguda. La CV-VHB fue positiva en 7 de 14 pacientes al momento del trasplante, con una mediana de $2832 \mathrm{UI} / \mathrm{mL}$ (609-19762 $\mathrm{UI} / \mathrm{mL}$ ). El 94\% de los pacientes recibía antiviral antes del trasplante, tiempo promedio de $17,9 \pm 17,7$ meses en los casos de infección crónica versus $0,28 \pm 0,31$ meses en los casos de insuficiencia hepática aguda. El entecavir fue el antiviral más frecuentemente utilizado (67\%).
Tabla 1. Características basales de la población

\begin{tabular}{|c|c|}
\hline Variable & $\begin{array}{l}\text { Total } \\
(\mathrm{n}, \%)\end{array}$ \\
\hline Hombre & $14(78,7 \%)$ \\
\hline Edad en años, promedio, DE & $52,6 \pm 11,28$ \\
\hline Comorbilidades & $15(83 \%)$ \\
\hline Hipertensión arterial & $6(40 \%)$ \\
\hline Diabetes mellitus & $4(26,6 \%)$ \\
\hline Cardiopatía & $2(13,3 \%)$ \\
\hline \multicolumn{2}{|l|}{ Indicación del trasplante } \\
\hline Hepatocarcinoma & $9(50 \%)$ \\
\hline Cirrosis & $4(22,2 \%)$ \\
\hline Insuficiencia hepática aguda & $4(22,2 \%)$ \\
\hline Tumor fibrolamelar & $1(5,6 \%)$ \\
\hline HBsAg pretrasplante & $18(100 \%)$ \\
\hline $\begin{array}{l}\text { HBeAg positivo pretrasplante (evaluado en } 12 \\
\text { pacientes) }\end{array}$ & $5(41,6 \%)$ \\
\hline $\begin{array}{l}\text { Carga viral VHB positiva pre-TH, evaluado en } \\
14 \text { pacientes }\end{array}$ & $7(50 \%)$ \\
\hline $\begin{array}{l}\text { Mediana de carga viral VHB al TH, mediana } \\
\text { p25-75 en unidades internacionales/mL }\end{array}$ & $2832(609-19762)$ \\
\hline Uso de antivirales previo al trasplante & $17(94,4 \%)$ \\
\hline Antiviral usado pretrasplante & $18(100 \%)$ \\
\hline Lamivudina & $6(33,3 \%)$ \\
\hline Entecavir & $12(66,7 \%)$ \\
\hline \multicolumn{2}{|c|}{$\begin{array}{l}\text { Tiempo de uso de antiviral previo al trasplante, mediana, rango, en } \\
\text { meses }\end{array}$} \\
\hline Insuficiencia aguda & $0,5(0,94-0,6)$ \\
\hline Insuficiencia crónica & $19(1,75-27)$ \\
\hline Coinfección con VHC & 0 \\
\hline Coinfección con VIH & $1(5 \%)$ \\
\hline Complicaciones en el pos-TH & $15(83,3 \%)$ \\
\hline Sepsis pos-TH & $4(26,6 \%)$ \\
\hline Infección oportunista & $4(22,2 \%)$ \\
\hline Trombosis vascular & $2(13,3 \%)$ \\
\hline Sangrado postoperatorio & $2(11,1 \%)$ \\
\hline Rechazo celular & $2(13,3 \%)$ \\
\hline
\end{tabular}

DE: desviación estándar; HBeAg: antígeno e del VHB; HBsAg: antígeno de superficie del VHB; TH: trasplante hepático; VHB: virus de la hepatitis B; VHC: virus de la hepatitis C; VIH: virus de la inmunodeficiencia humana.

\section{Profilaxis con inmunoglobulina}

De los 18 pacientes, 16 recibieron $\mathrm{HBIG}$ por $23,68 \pm 14,80$ meses acompañada de lamivudina o entecavir en el $27,7 \%$ y $72,2 \%$ de los casos, respectivamente. De los 18 pacientes, 
2 recibieron $\mathrm{HBIG}$ solo los primeros 7 días del trasplante y continuaron con entecarvir (tabla 2).

Tabla 2. Tratamiento recibido en 18 pacientes durante el seguimiento

\begin{tabular}{lc}
\hline \multicolumn{1}{c}{ Variable } & $\begin{array}{c}\text { Total } \\
(\mathbf{n}, \%)\end{array}$ \\
\hline Antiviral postrasplante & $18(100 \%)$ \\
Lamiduvina & $5(27,7 \%)$ \\
Entecavir & $13(72,2 \%)$ \\
HBIG & $18(100 \%)$ \\
1 semana & $2(11 \%)$ \\
Más de 1 semana & $16(88 \%)$ \\
Promedio de uso de la HBIG, promedio, DE, en meses & $23,68 \pm 14,80$ \\
\hline
\end{tabular}

DE: desviación estándar; HBIG: inmunoglobulina antihepatitis B.

\section{Seguimiento serológico}

Se encontró seroconversión del HBeAg en 11 de 14 pacientes evaluados. Durante el seguimiento, 2 de 18 pacientes $(11,1 \%)$ tuvieron positividad para el HBsAg. En uno de ellos se observó una recaída de la infección a los 41 meses postrasplante y al tercer mes de la suspensión de la HBIG, con una carga viral de 464977202 copias/mL a pesar del uso continuado de lamivudina. Este paciente había recibido lamivudina durante los 19 meses previos al trasplante. Después de detectarse la recaída, se modificó la terapia por entecavir sin respuesta, lo que motivó un nuevo cambió por tenofovir, con lo que se logró un mejor control sobre la carga viral y mejor función del injerto. En 2 de 18 casos hubo recurrencia del hepatocarcinoma no explicada por replicación viral en el postrasplante (tabla 3). La tasa de recaída global fue del 5,5\% (1 de 18 pacientes).

\section{Disfunción del injerto}

Hubo 8 casos de disfunción del injerto durante el seguimiento: 2 , por estenosis de la vía biliar; 1 , por hepatotoxicidad; 2 , por rechazo celular agudo leve; 1 , por hepatitis crónica periportal; 1 por recaída de la infección por el virus B; y 1 , por recaída de un tumor fibrolamelar. Todos los casos de disfunción fueron transitorios, salvo en el paciente con tumor fibrolamelar, en quien progresó la enfermedad. La disfunción global del injerto fue del 10\% a 33 meses (figura 1) y no hubo ningún fallecimiento durante el seguimiento.

\section{Reacciones adversas}

No se registraron eventos adversos relacionados con la terapia.
Tabla 3. Desenlaces en 18 pacientes sometidos a trasplante hepático con infección por virus de hepatitis B

\begin{tabular}{lc}
\hline \multicolumn{1}{c}{ Variable } & $\begin{array}{c}\text { Total } \\
\text { (n, } \%)\end{array}$ \\
\hline Tiempo de seguimiento en meses, mediana, rango & $\begin{array}{c}43,27 \\
(14,7-65,2)\end{array}$ \\
Seroconversión del HBeAg, evaluada en 14 & $11(78,5 \%)$ \\
pacientes & \\
HBsAg positivo en el pos-TH & $2(11,1 \%)$ \\
Carga viral positiva en el pos-TH evaluada en 15 & $1(6,6 \%)$ \\
pacientes & \\
Recurrencia & $1(5,5 \%)$ \\
Recurrencia de hepatocarcinoma & $2(11 \%)$ \\
Pérdida del injerto o retrasplante & $0(0)$ \\
Disfunción del injerto & $8(44,4 \%)$ \\
Recuperación total de la disfunción & $7(87,5 \%)$ \\
Mortalidad & 0 \\
\hline
\end{tabular}

HBeAg: antígeno e del VHB; HBsAg: antígeno de superficie del VHB; TH: trasplante hepático.

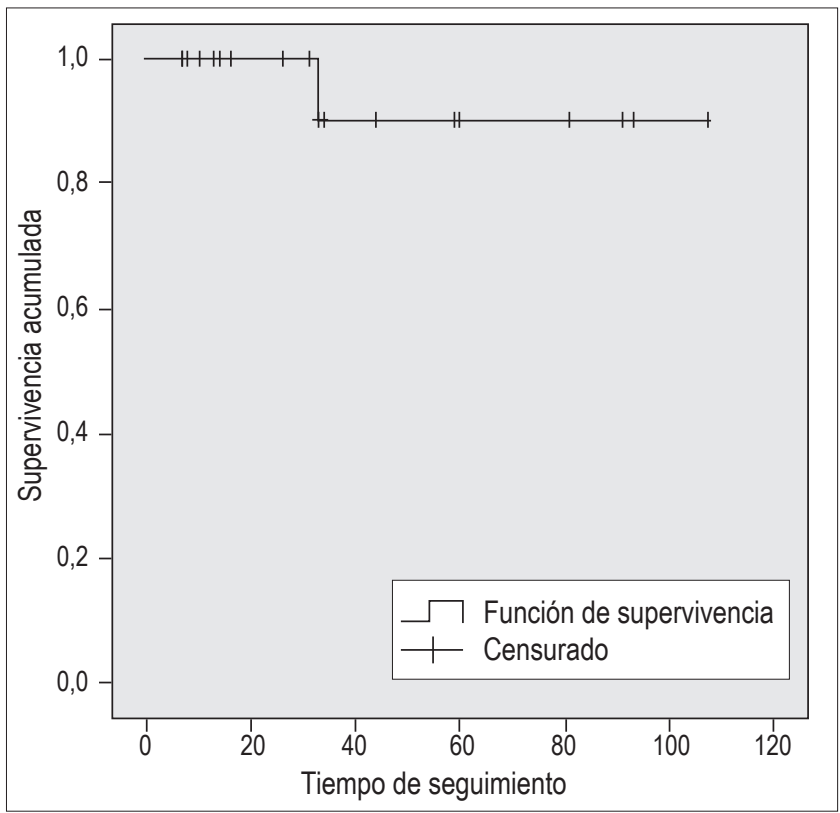

Figura 1. Disfunción del injerto durante el seguimiento. Curva de Kaplan-Meier.

\section{Costos}

Dado que las tasas de recurrencia reportadas en este estudio son similares a las reportadas en estudios que utilizaron dosis altas de HBIG, podría suponerse que ambos protocolos coinciden en efectividad, pero difieren ampliamente en costo. Aclaramos que no es un estudio de costo-efectividad y que solo son datos observacionales. 


\section{DISCUSIÓN}

En la actualidad, el trasplante hepático es la mejor alternativa para pacientes con complicaciones crónicas de la infección por VHB (11, 12). A principios de 1990, la profilaxis con HBIG en dosis altas se convirtió en la mejor alternativa terapéutica para disminuir la reinfección del injerto y la mortalidad (4). Markowitz (13) optimizó la profilaxis combinando HBIG con lamivudina, pero el costo de las dosis altas de inmunoglobulina ha sido un factor limitante y ha dado lugar al surgimiento de protocolos con dosis más bajas; sin embargo, se ha dudado de la capacidad para generar anticuerpos neutralizantes y evitar la recurrencia con dosis bajas.

McCaughan y colaboradores usaron dosis bajas de HBIG en 9 pacientes a 17 meses postrasplante sin ninguna recaída (14). Angus y colaboradores usaron dosis bajas de HBIG en 32 pacientes sin recurrencias a 18 meses (10). Finalmente, en 2007, Gane y colaboradores emplearon dosis bajas de HBIG con lamivudina en una cohorte australiana y neozelandesa, y encontraron una tasa de reinfección del $4 \%$ a 5 años, con una sobrevida del $82 \%$ a 1 año y del $88 \%$ a 5 años (9), similar a lo reportado con protocolos de dosis altas. Reportamos la experiencia utilizando un protocolo de dosis bajas de HBIG combinado con lamivudina o entecavir en pacientes trasplantados, con hepatitis $\mathrm{B}$, en un centro de referencia de trasplante hepático en Colombia; los resultados concuerdan con los reportes en otras series, con una tasa de recurrencia de la infección solo del 5,5\% (1 de 18 pacientes recurrió). En esta paciente que recayó se desconocía la carga viral al momento del trasplante, aunque había recibido 19 meses de lamivudina en monoterapia previamente. La recaída ocurrió 3 meses después de suspender la HBIG, por lo que sospechamos resistencia al análogo nucleósido; sin embargo, también tuvo recaída con el entecavir y mostró mejoría solo después de iniciar el tenofovir y reducir la inmunosupresión controlando la viremia.

La mayoría de los pacientes negativizaron el HBsAg durante el seguimiento, por lo que se considera un buen parámetro de seguimiento. Solo 1 paciente tuvo $\mathrm{HBsAg}$ positivo durante el seguimiento, sin que se correlacionara con la positividad de la carga viral. No documentamos efectos adversos relacionados con la aplicación de HBIG así como en las otras cohortes, donde el reporte de eventos adversos con dosis bajas de HBIG es infrecuente. Se requieren más reportes similares a este que apoyen el uso de inmunoglobulina en dosis bajas como alterativa de profilaxis en países de bajos ingresos, debido a que se presumen tiempos prolongados o indefinidos de profilaxis dada la escasa respuesta a la vacunación en esta población $(15 \%$ a 1 año y $8 \%$ a 2) (15). A este respecto, se han publicado estudios que proponen un uso por tiempo limitado de
HBIG combinado con un antiviral de alta barrera genética (tenofovir o entecavir) $(13,16,17)$, e incluso esquemas libres de HBIG (18).

Choudhary y colaboradores reportaron un estudio que utilizó HIBG por 12 meses seguido de tenofovir o entecavir (19), que mostró una tasa de recaída del 4,3\% a una mediana de seguimiento de 43 meses (12-117). En el mismo sentido, Tanaka y colaboradores encontraron una tasa del 0\% de recaídas a 29 meses utilizando HBIG durante 12 meses, seguido de tenofovir combinado con lamivudina en el postrasplante (20). También se describen protocolos libres de HBIG con recaídas del 1,26\% a 26 meses usando entecavir, en monoterapia una vez se haya controlado la carga viral (21). El tiempo promedio de uso de HBIG en nuestros pacientes fue de $23 \pm 14$ meses; esto obedece a que los pacientes trasplantados en los primeros años solían usar HBIG indefinidamente, pero, en los últimos años, con el advenimiento de nuevos antivirales, se optó por acortar el tiempo de HBIG, en especial cuando se lograba la seroconversión de los HBeAg y HBsAg (22). Hay un aumento progresivo en el uso de análogos de nucleósidos en monoterapia o terapia combinada con otro análogo en profilaxis, que disminuye cada vez más el uso de la HBIG; sin embargo, se requieren estudios a largo plazo acerca de la seguridad de los esquemas libres de HBIG. Mientras tanto, en muchos centros de trasplante a nivel mundial siguen usando HBIG como el tratamiento óptimo para prevenir la reinfección.

Este estudio tiene varias limitaciones, entre ellas: el carácter retrospectivo; el desconocimiento de la carga viral al momento del trasplante en 4 pacientes, por lo que no es posible hacer asociaciones entre CV-VHB pretrasplante y recaída; la falta de evaluación sistemática con carga viral en el postrasplante. Sin embargo, se hizo un seguimiento extenso que permitió evaluar la mortalidad a más de 3 años y la función del injerto a largo plazo. No realizamos análisis de costo-efectividad, aunque la aproximación de costos nos permite inferir que a menor costo usando dosis bajas de HBIG se obtienen resultados similares a los reportados con la administración de dosis altas.

Mientras avanzan los estudios de profilaxis libre de HBIG y se obtiene suficiente seguridad a largo plazo, la profilaxis con dosis bajas de HBIG en prevención de recurrencia de infección postrasplante sigue siendo de utilidad.

\section{Conflictos de interés}

Los autores declaran no tener ningún conflicto de intereses.

\section{Apoyo financiero}

Este trabajo no contó con apoyo financiero de ninguna entidad. 


\section{REFERENCIAS}

1. European Association for the Study of the Liver. EASL clinical practice guidelines: Management of chronic hepatitis B virus infection. J Hepatol. 2012;57(1):167-85.

2. Cubides VI, Suárez CY, Quintero PA. Epidemiología e historia natural de la hepatitis B. Rev Col Gastroenterol. 2009;24(1):4-12.

3. Tolosa-Pérez N. Protocolo de vigilancia en salud pública. Hepatitis B, C y coinfección hepatitis B-Delta. Bogotá: Instituto Nacional de Salud; 2014.

4. Lok AS. Prevention of recurrent hepatitis B post-liver transplantation. Liver Transplant. 2002;8(10 Supl 1):67-73.

5. Mutimer D. Review article: hepatitis B and liver transplantation. Aliment Pharmacol Ther. 2006;23(8):1031-41.

6. Marzano A, Salizzoni M, Debernardi-Venon W, et al. Prevention of hepatitis B virus recurrence after liver transplantation in cirrhotic patients treated with lamivudine and passive immunoprophylaxis. J Hepatol. 2001;34(6):903-10.

7. Grellier L, Mutimer D, Ahmed M, et al. Lamivudine prophylaxis against reinfection in liver transplantation for hepatitis B cirrhosis. Lancet. 1996;348(9036):1212-5.

8. Mutimer D, Dusheiko G, Barrett C, et al. Lamivudine without HBIg for prevention of graft reinfection by hepatitis B: longterm follow-up. Transplantation. 2000;70(5):809-15.

9. Gane EJ, Angus PW, Strasser S, et al. Lamivudine plus lowdose hepatitis B immunoglobulin to prevent recurrent hepatitis B following liver transplantation. Gastroenterology. 2007; 132(3):931-7.

10. Angus PW, McCaughan GW, Gane EJ, et al. Combination low-dose hepatitis B immune globulin and lamivudine therapy provides effective prophylaxis against posttransplantation hepatitis B. Liver Transplant. 2000;6(4):429-33.

11. Markowitz JS, Martin P, Conrad AJ, et al. Prophylaxis against hepatitis B recurrence following liver transplantation using combination lamivudine and hepatitis B immune globulin. Hepatology. 1998;28(2):585-9.

12. Xi ZF, Xia Q. Recent advances in prevention of hepatitis $B$ recurrence after liver transplantation. World J Gastroenterol. 2015;21(3):829-35.
13. Ahn J, Cohen SM. Prevention of hepatitis B recurrence in liver transplant patients using oral antiviral therapy without long-term hepatitis B immunoglobulin. Hepat Mon. 2011;11(8):638-45.

14. McCaughan GW, Spencer J, Koorey D, et al. Lamivudine therapy in patients undergoing liver transplantation for hepatitis B virus precore mutant-associated infection: high resistance rates in treatment of recurrence but universal prevention if used as prophylaxis with very low dose hepatitis $B$ immune globulin. Liver Transplant.1999;5(6):512-9.

15. Togashi J, Akamastu N, Sugawara Y, et al. One-year extended, monthly vaccination prophylaxis combined with hepatitis B immune globulin for hepatitis B after liver transplantation. Hepatol Res. 2016;46(3):E51-9.

16. Varghese J, Sachan D, Reddy MS, et al. Hepatitis B immunoglobulin prophylaxis after liver transplantation: experience in a tertiary transplant centre. J Clin Exp Hepatol. 2014;4(3):209-13.

17. Lim YS, Han S, Heo NY, et al. Mortality, liver transplantation, and hepatocellular carcinoma among patients with chronic hepatitis B treated with entecavir vs lamivudine. Gastroenterology. 2014;147(1):152-61.

18. Teperman LW, Poordad F, Bzowej N, et al. Randomized trial of emtricitabine/tenofovir disoproxil fumarate after hepatitis B immunoglobulin withdrawal after liver transplantation. Liver Transplant. 2013;19(6):594-601.

19. Choudhary NS, Saraf N, Saigal S, et al. Low-dose short-term hepatitis B immunoglobulin with high genetic barrier antivirals: the ideal post-transplant hepatitis B virus prophylaxis? Transpl Infect Dis. 2015;17(3):329-33.

20. Tanaka T, Renner EL, Selzner N, et al. One year of hepatitis $\mathrm{B}$ immunoglobulin plus tenofovir therapy is safe and effective in preventing recurrent hepatitis B post-liver transplantation. Can J Gastroenterol Hepatol. 2014;28(1):41-4.

21. Fung J, Cheung C, Chan SC, et al. Entecavir monotherapy is effective in suppressing hepatitis B virus after liver transplantation. Gastroenterology. 2011;141(4):1212-9.

22. Jeong SW, Choi Y, Kim JW. Management of viral hepatitis in liver transplant recipients. Clin Mol Hepatol. 2014;20(4):338-44. 